\title{
Review of soft tissue augmentation in the face
}

This article was published in the following Dove Press journal:

Clinical, Cosmetic and Investigational Dermatology

28 August 2009

Number of times this article has been viewed

\section{James Newman}

Facial Plastic Surgery, Stanford University Medical Center, Palo Alto, CAFacial Plastic Surgery, Stanford University Medical Center,

Palo Alto, CA, USA
Correspondence: James Newman Director of Premier Plastic Surgery Clinic Palo Alto, CA, USA

Email newman_md@hotmail.com
Abstract: A primary pillar of facial rejuvenation is the replacement of soft tissue atrophy via a variety of augmentation techniques. The techniques can be classified into three categories, skeletal onlay grafts, subcutaneous volumizers, and dermal fillers. While onlay grafts and subcutaneous volumizers have the most persistent results, the emergence of improved dermal fillers in the past 5 years has become increasingly popular. An accurate diagnosis of the level(s) of soft tissue atrophy in the face needs to be made prior to selection of the category or combination of techniques. In the younger patient, the selection of a dermal filler or combination of fillers can be adequate for treatment. A comparison of the composition and characteristics of the available dermal fillers are discussed in detail to assist the clinician in understanding the actual mechanism of soft tissue augmentation. In the more advanced aging face, a combination of the three categories may be necessary to produce optimal results. Just as dermal fillers have become more differentiated to increase their longevity, the non-injectible long-lasting implants are becoming more developed to mimic accurate viscoelastic properties of the facial soft tissues. All three classes of augmentation techniques can provide patients with very satisfactory results as part of overall facial rejuvenation.

Keywords: soft tissue, dermal fillers, facial implants, facial augmentation

As the face ages, wrinkles, grooves, and ptotic tissue become more prominent. Superficial wrinkles are largely due to photo damage and resulting solar elastosis. This is characterized by loss of collagen mass in the epidermal-dermal junction and an increased array of elastin whirls in the deeper dermis. Repeated muscle action produces prominent wrinkles and creases in the mimetic areas of facial skin such as the glabella, periorbital skin, nasolabial creases, and perioral skin. Grooves appear deeper in the nasolabial and marionette zones with the additional feature of fat atrophy. As a result of the loss of fat volume, the static suspensory ligaments become more lax and the face takes on attributes of ptotic jowls, ptotic malar mounds, and nasolabial folds. Skeletal changes resulting in decreased height of the maxilla and the mandible occur in the later decades of life (6th-8th decade) and accentuate the above findings.

Facial rejuvenation requires an accurate diagnosis of the above findings, and therapies are directed at correcting multiple layers. There are four pillars of facial rejuvenation: 1) ensuring adequate skeletal framework and support, 2) tightening and repositioning of the investing musculofascial aponeurotic system of the face and neck (galea, superficial muscular aponeurotic system [SMAS], and platysma), 3) replacement 
of soft tissue volume loss, and 4) redraping and removal of excess skin.

This paper focuses on the third pillar of facial rejuvenation and reviews the choices available for soft tissue enhancement and replacement. Present-day surgeons have a number of choices for facial augmentation which can be tailored according to the layer(s) which needs augmentation. The options for rejuvenation can be classified as skeletal onlay grafts, subcutaneous volume enhancers, and dermal fillers. One can also think of the simple classification according to depth. The skeletal onlay grafts work from the bone upward, the dermal grafts work from the dermis downward, and the subcutaneous fillers are in between the two. Dermal fillers and subcutaneous volume enhancers have enjoyed the greatest degree of development and differentiation because they are administered in an office-based setting. Each of the three options for volume enhancement will be discussed with clinical examples of each.

\section{Skeletal onlay grafts}

Skeletal onlay grafts remain one of the most reliable and safe options for soft tissue augmentation. Although this is an indirect method of soft tissue augmentation, the results of enhancing the overlying tissues are indisputable. Much experience has been gained from the use of custom carved grafts for congenital malar and mandibular hypoplasia and the beneficial repositioning of the facial soft tissues that brings improved facial harmony. Custom preformed grafts are now manufactured from materials such as silastic, expanded polytetrafluoroethylene (ePTFE), and Porex. ${ }^{1}$

These implants require an incision and dissection either above or below the periosteum. When the implant is placed, it pushes all the overlying soft tissue and skin outward and in essence serves as a deep soft tissue filler. These implants most commonly involve the chin, mandibular angle, malar complex, and nasal dorsum. Skeletal onlay grafts are commonly used to address soft tissue volume loss from the adipose layers and restore three-dimensional convexity to the midface. Examples of onlay grafts enhancing soft tissue in the chin and midface are shown in Figure 1.

The placement of skeletal onlay grafts are surgical procedures which involve sterile fields and adequate knowledge of anatomy to preserve the motor and sensory nerves. These implants are typically stabilized with either bone fixation or suture/bolster combinations. Patients are counseled to expect edema and temporary anesthesia for 3 weeks and capsule maturation after 6 months. There are no clinically significant alterations to bone volume resulting
A

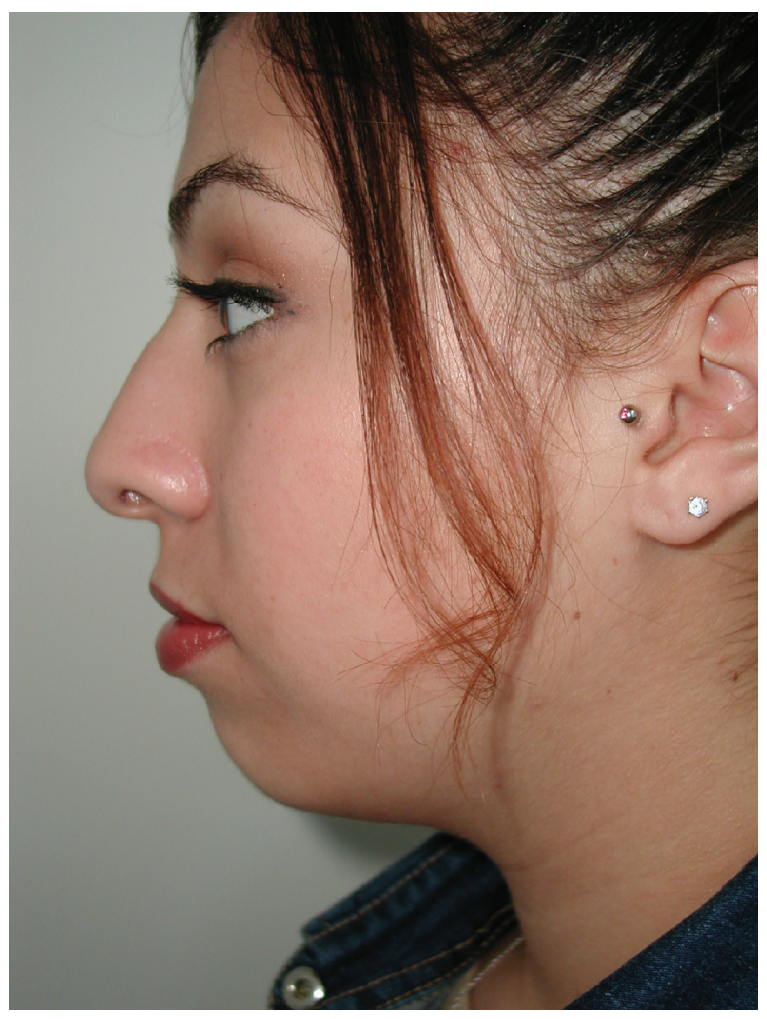

B

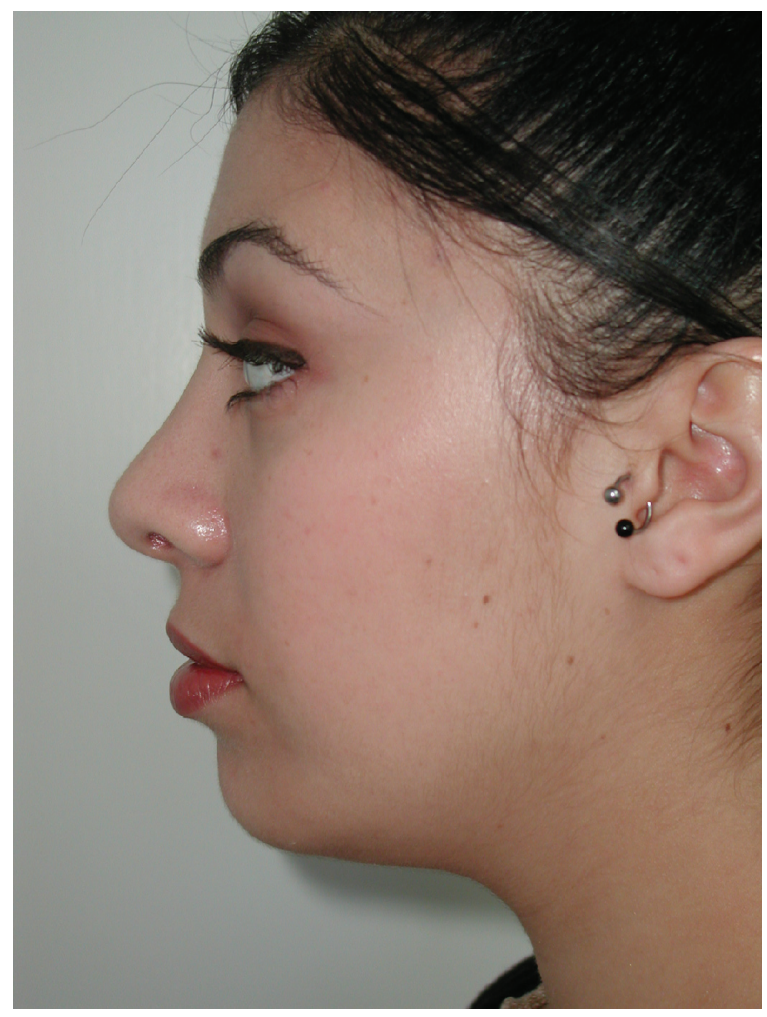

Figure IBefore (A) and after (B) example of soft tissue augmentation with silastic chin onlay graft. 
in bone loss or soft tissue loss as these implants have been in widespread use for the past 30 years. These types of soft tissue implants are reversible if there is any need to remove them. The most challenging feature of skeletal onlay grafting lies not with the technique of placement but in the selection of the proper size which matches both the patient and physician's esthetic goals. Fortunately with computeraided design, a good selection of three-dimensional shapes exist with corresponding sizers which allow for more accurate selection.

Further refinements in soft tissue augmentation can be made with injectible fillers and/or autogenous fat to account for further aging that occurs. Additional dermal fillers such as Sculptra ${ }^{\circledR}$ (Sanofi-Aventis, Summit, NJ, USA), hyaluronic gels, and Radiesse ${ }^{\circledR}$ (Bioform Medical, San Mateo, CA, USA) have been placed over in situ silastic implants without complications for the past 3 years. Some surgeons prefer exclusive use of autogenous materials for augmentation. These grafts can also be custom carved from the outer calvarium, ribs, and iliac crest. For smaller onlay grafts particularly in the nasal dorsum, septal cartilage, ear cartilage, and irradiated rib cartilage are also some options. These materials have stood the test of time and are generally preferred when alloplastic implants are not practical because of concerns about the health of overlying soft tissues. They increase the duration of the procedures and have some added morbidity because of the need for a donor site. The skeletal onlay graft remains the most predictable and permanent option for soft tissue enhancement of the face.

\section{Subcutaneous volume enhancers}

Autologous fat is the cornerstone of facial volume filling for the surgeon in the operating room and should be considered in almost every case where generalized soft tissue loss is noted in the aging face of immunocompetent patients. ${ }^{2}$ Success with fat grafting still varies among practitioners over the past few decades. The key factors which seem to affect overall success include harvest methods, placement techniques, and choice of recipient site. It is an accepted fact that low negative pressure suction with manual syringes offers the least traumatic method of fat harvesting. Typically $3-\mathrm{mL}$ to 10-mL syringes are used with 16-gauge needles or cannulas to harvest subcutaneous fat from the abdomen, lateral thighs, gluteal areas, or medial knees. Gentle handling of fat with some sort of separation from oils and blood can be accomplished through gravity decantation or centrifuging. Transfer of the harvested fat to 1-mL syringes and blunt cannulas of various curves and sizes offers precise placement of small
0.1-mL aliquots of fat. Using blunt cannulas, low pressure and typically depositing on withdrawal of the cannula allow for more precise placement of fat pearls at various levels including intramuscular levels.

The most common areas of the face which are treated with autologous fat grafts include the nasolabial groove, marionnete lines, midface, and lips. Autologous fat performs best in the midface area considering the longevity compared to other more mobile areas such as lips and marionette grooves. Fat also remains the filler of choice in combination with aging face surgery. These patients are generally in need of more extensive volume replacement and add little additional morbidity to the expected bruising and edema typically encountered in the postoperative surgical recovery period. The challenging areas for successful fat grafting include the nasojugal area and lower lid creases. These areas are the most technique dependent for obtaining smooth results and greater complications arise with fat grafting in these areas. Strategies to minimize complications in these areas include the use of small cannulas which allow ultrafine pearls of fat placement and vertical and horizonal vector placement paths to avoid clumping. ${ }^{2}$

There are patients in whom fat grafting is not realistic, such as patients with low body fat, eg, long distance runners, those in advanced age, and those who have HIV-associated lipodystrophy. These patients have been able to withstand large volumes and concentrations of synthetic injectible volumizers such as calcium hydroxylapatite (Radiesse) and poly-L-lactic acid (Sculptra). Both Radiesse and Sculptra are approved in the US by the FDA for correction and restoration of facial fat loss (lipoatrophy). These patients can take high volumes of fillers, typically 3 to 4 times the amount used in the aging-face population. These fillers will be discussed in more detail.

Subcutaneous augmentation of the lips is one of the most common requests, not only in the aging population but also in young adults desiring enhanced lip volume. In addition to fat, autologous SMAS fascia has been used to augment subcutaneous tissue in the nasolabial groove and lip with variable results. $^{3}$ There is no filler or implant currently approved by the FDA for lip enhancement. The hyalurons are most commonly used off-label, with satisfactory but temporary results. The difficulty in enhancing the lips relates to the irregular nature of the mucous glands in the lips and the temporary nature of the hyaluronic gels.

Prior attempts of using alloplastic materials such as tubular ePTFE have been suboptimal because of complete tissue ingrowth and hardening of some of these implants. Improvements in the designs have led to better tolerance but have not been able to mimic the softness of hyaluronic 
gel until the development of saline-filled implants. (Fulfil ${ }^{\circledR}$; Evera Medical, Foster City, CA, USA). The subcutaneous lip implantation is done under local anesthesia and it mimics the soft tissue of the lip extremely well. ${ }^{4}$ The implant is composed of a dual-layer membrane which contains the saline. These implants are unique because they include a dual layer, an inner elastic membrane, and outer microscopic ePTFE membrane with a slip plane in between, allowing the implant to stretch. By allowing the implant to stretch in vivo, this becomes the first alloplastic implant which more closely mimics the viscoelastic tissues of the soft tissues of the lips and face. These implants are currently being evaluated in a controlled FDA trial to assess their cosmetic efficacy for lip augmentation. An example of one of the Fulfil implants is shown in Figure 2 and a patient example is shown in Figure 3. These alloplastic implants offer a potential permanent method of subcutaneous augmentation in one of the more challenging areas of the face. Disadvantages include the fact that the procedure comes with a short duration of swelling that the patient must plan for and the possible risk of infection with an implant.

\section{Dermal fillers}

The ideal dermal filler is one that is biocompatible, predictable, adjustable to the anatomy of the patient, long-lasting, reversible, and natural in appearance. While no single filler possesses all of these characteristics, a systematic review will highlight the ingredients of each filler and how each one behaves clinically. Dermal filler use began in the mid 1980s and has since grown, with no fewer than 10 new fillers in the US market. Dermal fillers have become the cornerstone of facial filling in the

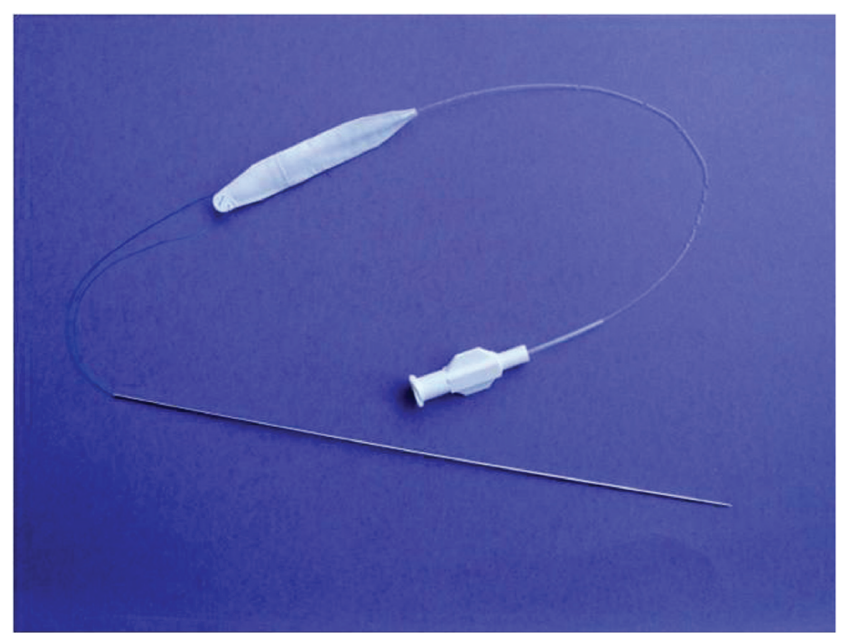

Figure 2 Subcutaneous volumizer Fulfil implant prior to placement. Guide needle and fill tube shown. Implant is inflated with saline to expand surrounding soft tissue. office setting. A summary of 12 dermal fillers is shown in Table 1 with a simple classification system based on the main active component.

\section{Collagen}

Bovine collagen was the first filler available and included the formulations of Zyderm ${ }^{\circledR}$ and Zyplast ${ }^{\circledR}$ (Allergan, Irvine, CA, USA). These products ranged from 35 to $65 \mathrm{mg} / \mathrm{mL}$ bovine collagen and had $0.3 \%$ lidocaine. The cross-linked "plast" product was made with glutaraldehyde. A skin test was required to screen for the $1.5 \%$ to $3 \%$ incidence of delayed type hypersensitivity. Human collagen equivalents in dosing and cross-linking $\left(\right.$ Cosmoderm ${ }^{\circledR}$ and Cosmoplast ${ }^{\circledR}$; Allergan) were introduced to eliminate the need for allergy testing. All of the collagen products have clinical effects lasting from 1 to 4 months. The main clinical advantage of the human collagen products is their ability to correct the most superficial lines with smooth flow characteristics as their carrier is phosphate-buffered saline. The duration of clinical effects has not been able to reach that of the hyaluronic gels as demonstrated in a blinded comparative study against Zyplast. ${ }^{5}$

A porcine-derived collagen (Evolence ${ }^{\circledR}$; OrthoNeutogena, NJ, USA) was given market clearance in 2008 with clinical data showing good tolerability without need for a skin test. Evolence is composed of $35 \mathrm{mg} / \mathrm{mL}$ biodegradable type I porcine collagen at a physiologic $\mathrm{pH}$ that appears as a yellowish opaque gel supplied in a 1-mL syringe and 27-gauge needle. Clinical results with porcine collagen show greater duration than with the human- or bovine-derived collagens. This collagen is highly purified from porcine antigens and is cross-linked with D-ribose, making it more resistant to degradation. Currently the duration of effect in the nasolabial grooves is similar to that of hyaluronic gels. It has very smooth flow characteristics through the supplied 27-gauge needle, as its carrier is phoshphate-buffered saline. ${ }^{6}$

\section{Hyaluronans}

Hyaluronic acid (HA) is a polysaccharide and a normal component of most tissues including dermis. As a result of antigenicity and the inconvenience of skin testing for collagen-based fillers, much recent attention has been focused on HA. The first HA approved for use in the USA showed superior results compared to bovine collagen ${ }^{5}$ and began a wave of HA products for the dermal filler market. HA is a ubiquitous component of mammalian connective tissue. HA-based polymers offer excellent biocompatibility while providing the same structural and mechanical properties of 
A

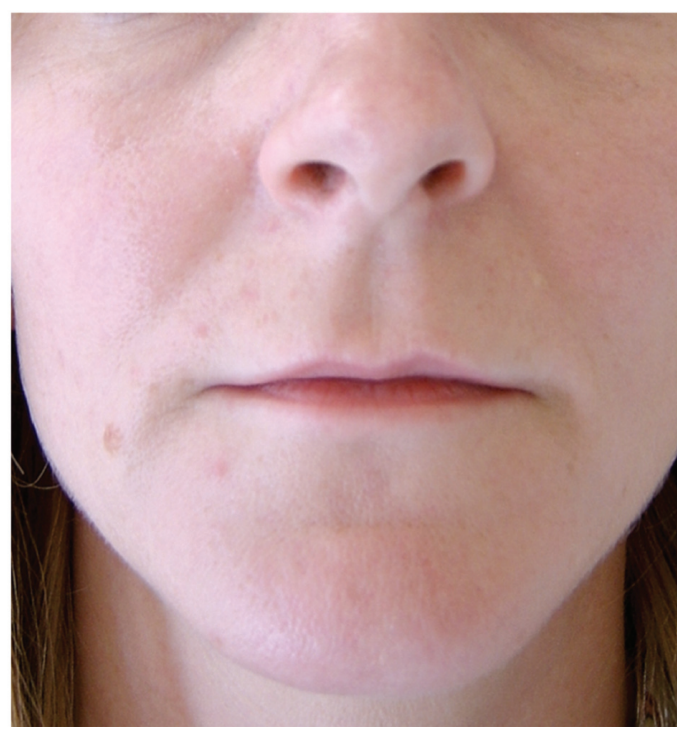

B

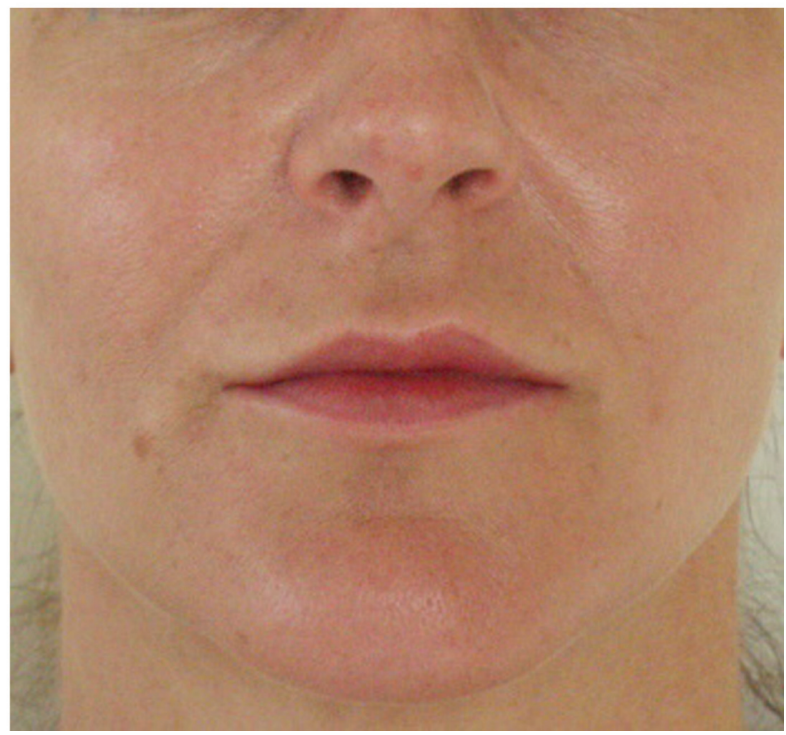

Figure 3 Before (A) and after (B) example of soft tissue augmentation of the lips with the Fulfil implant at 12 months.

normal subcutaneous tissue. HA, in its native form has a short life span. However, when cross-linked it persists significantly longer. Cross-linked hydrogels such as Juvederm ${ }^{\circledR}$ (Allergan), Elevess ${ }^{\circledR}$ (Anika Pharmaceutics, Waltham, MA, USA), Perlane ${ }^{\circledR}$, and Restylane ${ }^{\circledR}$ (both Medicis Aesthetics Scottsdale, AZ, USA), are among some of the currently FDA-approved products used for soft-tissue augmentation. All are uniquely cross-linked to give them an in vivo life span of 6 to 18 months.

Cross-linked derivatives have been shown to be well tolerated when injected into locations such as the skin and vocal folds. ${ }^{7}$ The use of HA is particularly attractive for soft-tissue augmentation, because it is hydrophilic and a normal extracellular component of skin. It is directly responsible for much of the tissue's function, and provides little in the way of a host immune response since it is conserved across species. Because of its tolerability profile, it can be used without skin testing and has a life-span that exceeds bovine collagen. In its native form, HA is readily metabolized by lymphatic clearance and ultimately degraded in the liver to carbon dioxide and water.

Factors that impact HA persistence include HA concentration, percentage of cross-linkage, type of cross-linking, its fluid retention (ie, water binding capacity), and injection technique. The two most important factors are the percentage of cross-linking and the water binding capabilitiy of the hyaluronic gel. When uncross-linked HA is added to water it produces a highly viscous liquid that would only last a few days in human skin. ${ }^{8}$ To improve the longevity, manufacturers use various agents to cross-link the HA. As the amount of cross-linking increases, the gel becomes more firm and feels more like a solid. As a result, the final proportion of cross-linked HA and the degree of cross-linking impact the physical characteristic of the final product. The water binding capacity or the hydrophilic nature allows the HA to create larger volumes relative to their mass. Recent studies suggest that increased concentrations of HA prolongs persistence. ${ }^{7}$ However the concentration alone is not the most important factor affecting tissue persistence but rather the extent of cross-linking of a particular product. Extent of cross-linking includes the degree of cross-linking and the percent of total product which is cross-linked. If all other factors were the same among $\mathrm{HA}$, the product with the higher degree of cross-linking experiences the least amount of degradation by enzymes and free radicals. ${ }^{8}$ Technique can play a role in longevity of the dermal filler. Injection into the deep dermis has been shown to increase de novo collagen synthesis, hypothesized to be the result of fibroblast stretching. ${ }^{9}$ Therefore as the HA is degraded, novel collagen synthesis replaces the HA resulting in longer-lasting correction.

One very important characteristic of HA products is the ability of clinicians to break down the cross-linking of each product with the use of an enzyme known as hyaluronidase. This enzyme breaks the cross-links by hydrolysis of the glucosamine and glucornic acid moiety. This results in the breakage of the cross-links and the three-dimensional structure 
of HA becomes resorbed within hours by the surrounding interstitial fluid. Hyaluronidase is available commercially in the US as Vitrase ${ }^{\circledR}$ (ISTA Pharmaceuticals) (200 units $/ \mathrm{mL}$ ), Amphadase $^{\circledR}$ (Amphastar Pharmaceuticals) (150 units $/ \mathrm{mL}$ ) or Hydase ${ }^{\circledR}$ (Prima Pharma) (150 units $/ \mathrm{mL}$ ). One note of caution is the possibility of allergic reaction with purified bovine testicular hyaluronidase or with preparations that contain metabisulfite. A simple skin test can be performed by injecting $0.02 \mathrm{~mL}$ ( 3 units) of a 150 unit $/ \mathrm{mL}$ solution. A positive reaction is seen within 5 minutes as a wheal with erythema and localized itching. Approximately 15-20 units of hyaluronidase can be injected directly into a pea-sized volume of HA and cause its dissolution within hours.

\section{Hyaluronic gels with lidocaine}

Elevess is an HA produced by Streptococcus equi (bacterial fermentation), cross-linked and suspended in a buffer solution at a concentration of $28 \mathrm{mg} / \mathrm{mL}$. ${ }^{10}$ Elevess contains $0.3 \%$ lidocaine $\mathrm{HCl}$ and sodium metabisulfite as an antioxidant. A skin test is not required. Elevess is a unique hyaluron because of the small amount of premixed lidocaine. It is a highly cross-linked product which has produced significant swelling in patients and requires a 27 -gauge needle instead of the the 30 -gauge needle supplied with the product. This product has not been in widespread use and has limited clinical information in the area of facial esthetic applications.

Prevelle Silk ${ }^{\circledR}$ (Genzyme Corporation) is another hyaluronic acid with $0.3 \%$ lidocaine. ${ }^{11}$ It contains $5.5 \mathrm{mg}$ of hyaluronic gel in a $0.75-\mathrm{mL}$ syringe. Due to its low concentration and limited cross-linking, it produces minimal swelling and also has a shorter duration of effect. It has some unique clinical advantages for situations in which a patient wants some immediate correction with minimum downtime. A good usage of this product is to highlight the vermillion border of the lips and to soften vertical perioral lip lines. The distribution rights for this product (Mentor Corporation) was acquired by Ortho Neutrogena in 2009 which distributes the Evolence collagen product for nasolabial grooves.

\section{Restylane}

Restylane is an HA gel generated by Streptococcus, chemically cross-linked with 1,4-butanediol diglycidyl ether (BDDE), stabilized and suspended in phosphate buffered saline at $\mathrm{pH} 7$ and concentration of $20 \mathrm{mg} / \mathrm{mL} .{ }^{12}$ Restylane is supplied in a glass syringe with a 30 -gauge needle. The restylane is $80 \%$ cross-linked, with its degree of cross-linking approaching $2 \%$. It also has a hardness value of approximately $400 \mathrm{~Pa}$ as compared to Perlane which has a value of 
A

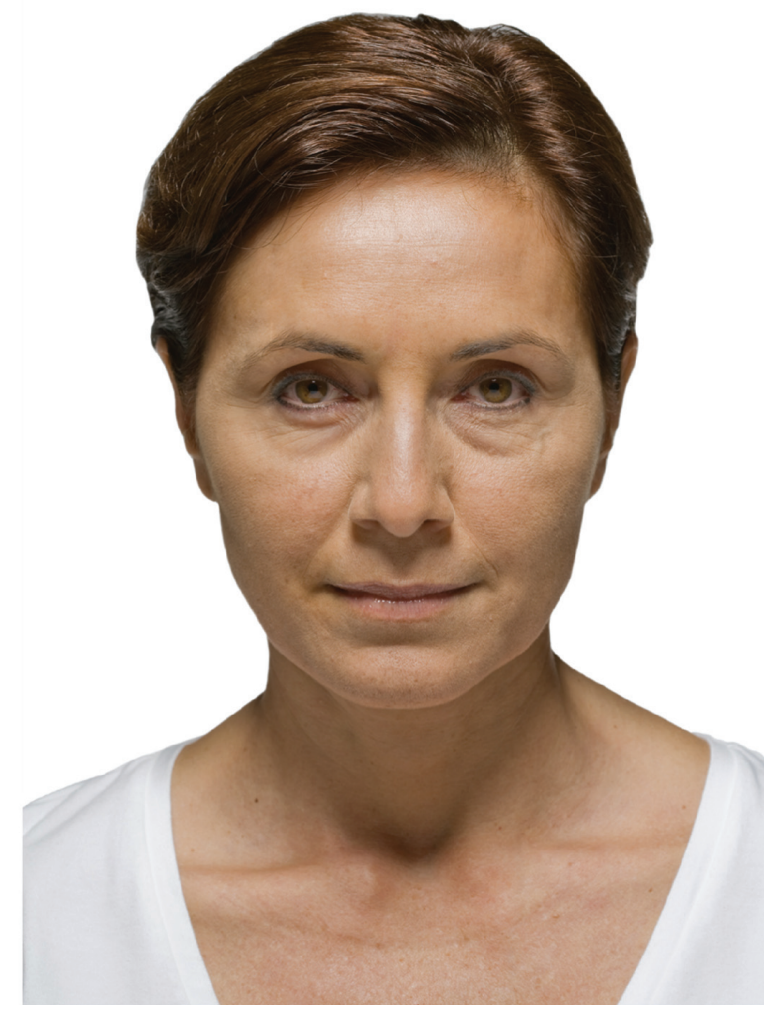

B

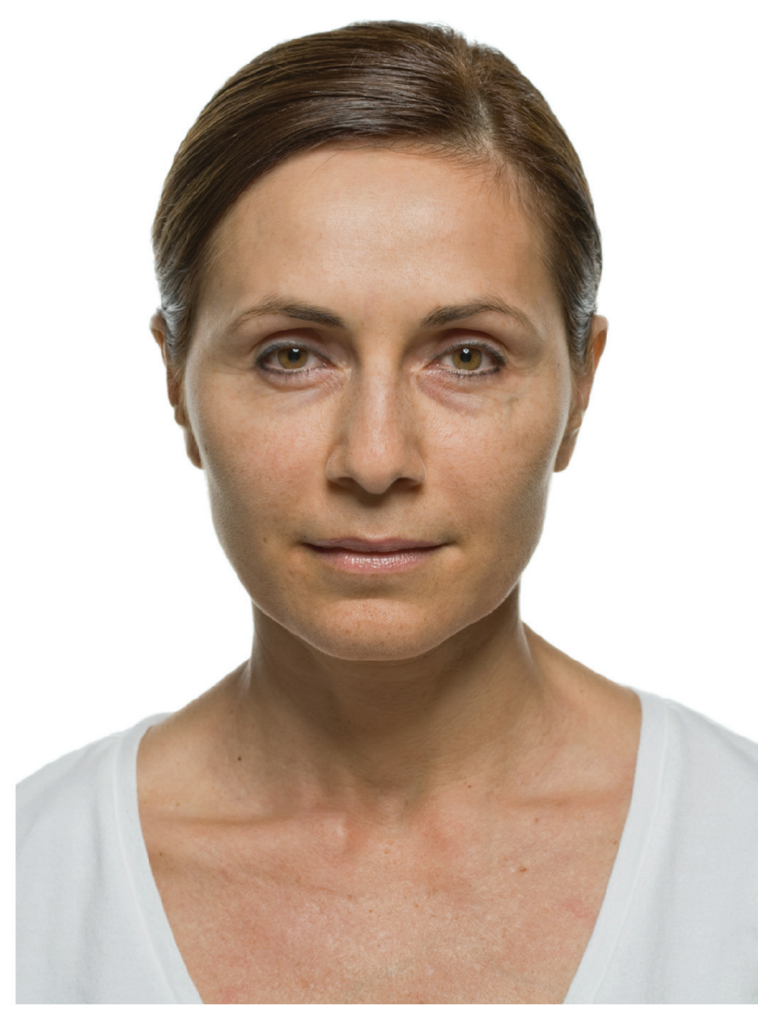

Figure 4 Before $(\mathbf{A})$ and after $(\mathbf{B})$ example of soft tissue augmentation of the nasolabial grooves with calcium hydroxlyapatite (Radiesse) at 6 months.

approximately $550 \mathrm{~Pa}$ when calculated at $5 \mathrm{~Hz}$. It was the first stabilized hyaluronic acid dermal filler of nonanimal source approved by the FDA in the United States. It has enjoyed widespread use in North America with over 1.4 million treatments as of 2007, and has an excellent safety profile, with skin sensitivity reactions noted in 1:10,000 cases. The Restlyane family of products in esthetic soft tissue use includes Perlane and Macrolane ${ }^{\mathrm{TM}}$ (Q-Med AB). All the products are manufactured by Q-med Corporation (Uppsala, Sweden) whose products are based on different particle sizes which can be filtered into different gels. The different-sized gel particles result in different exposures of the product to breakdown enzymes and free radicals. The larger-sized particles may also be placed deeper in soft tissues. The Restylane product contains 100,000 particles per mL, Perlane contains 10,000 particles per $\mathrm{mL}$ and Macrolane contains 1000 particles per $\mathrm{mL}$.

\section{Perlane}

Perlane is a sterile gel of hyaluronic acid generated by Streptococcus species of bacteria, chemically cross-linked with BDDE, stabilized, and suspended in phosphate buffered saline at $\mathrm{pH} 7$ and a concentration of $20 \mathrm{mg} / \mathrm{mL} .{ }^{13}$ This product is of the same concentration as Restylane and has the same amount and degree of cross-linking as Restylane. What makes this product different is the particle size which makes Perlane contain about 10,000 particles per $\mathrm{mL}$ compared to Restylane which has 100,000 particles per $\mathrm{mL}$. The largest fraction of gel particle size is between 940 and 1090 microns. The larger particle of Perlane is thought to increase stability of the product by reducing the surface area exposed to degradative forces (enzymes and free radicals). Macrolane is a product with even larger particle size which is available outside the US for use in body soft tissue augmentation.

\section{Juvederm}

Juvederm is an HA product derived from bacterial (Streptococcus equi) fermentation and has a concentration of $24 \mathrm{mg} / \mathrm{mL} .{ }^{14}$ It has two different configurations in the US (Juvederm Ultra and UltraPlus) and will soon have lidocaine incorporated into the product. All the Juvederm products contain the same concentration of HA and the differentiating factor is the amount of cross-linking 


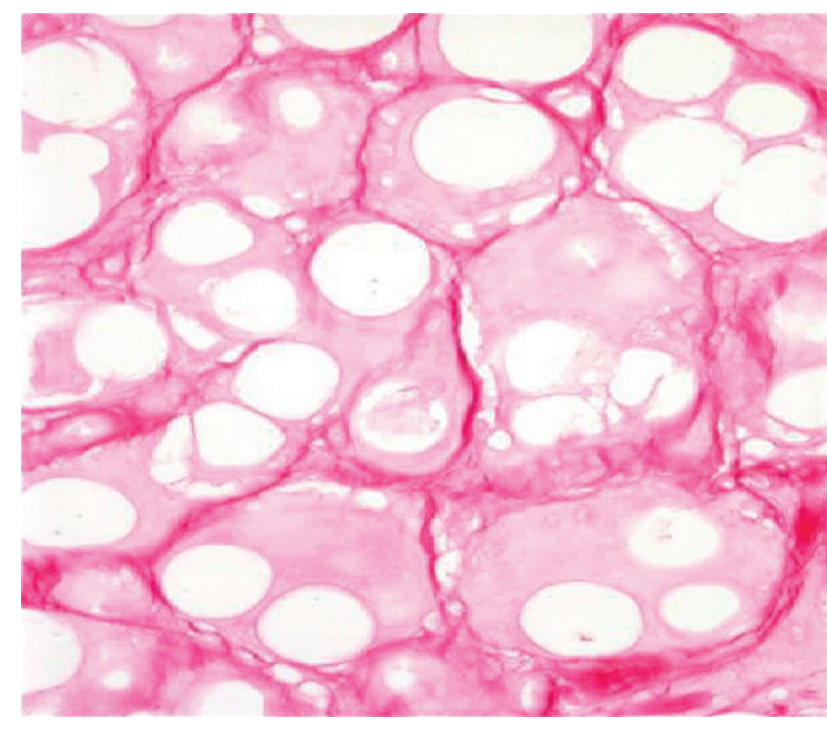

Figure $\mathbf{5}$ Histologic photomicrograph of calcium hydroxlyapatite with neocollagenesis at 16 months in a canine model.

in each product. The same cross-linking agent, BDDE, is utilized. The Juvederm Ultra product is $90 \%$ cross-linked with a degree of cross-linking at $6 \%$ and comes in a $0.8-\mathrm{mL}$ syringe with a 30 -gauge needle. Juvederm Ultra has a slightly smoother gel consistency compared to Juvederm Ultra Plus with a gel hardness measured at approximately $180 \mathrm{~Pa}$. The Ultra Plus has gel hardness of $200 \mathrm{~Pa}$ and is injected with a 27 -gauge needle compared to the Ultra product. The Ultra Plus has a higher degree of cross-linking of $8 \%$ when compared to Juvederm Ultra (6\%) and explains the longer duration of effect compared to the Ultra Juvederm product. The Juvederm family of products also seem to have higher hydrophilic properties compared to Restylane. The clinical significance of this observation has yet to be studied but the author's preference for off-label uses of the hyaluronic gels is for the use of Juvederm Ultra Plus for lip enhancement. In this location, the greater hydrophilic property plumps the lips and gives a softer feel. In the nasojugal area, Restylane is preferred because there is less edema and its greater firmness makes it easier to mold and avoid excess swelling for patients post-treatment.

\section{Potential complications of HA}

HA dermal fillers as a group are very well tolerated. Infection can occur but is rare. Hypersensitivity reactions are also uncommon, and may result from reaction to the crosslinking agent used to stabilize the HA. Occasionally HA can be palpated, or a blue-gray tinge can be seen in the area of injection. This can be the result of superficial injection allowing more water binding in the dermis which selectively reflects blue wavelength of light making it appear darker than the surrounding skin. Solutions to this problem can be addressed by camouflage with makeup, needle puncture, and massage of excess gel from the dermis or injection of hyaluronidase. Injection technique can lead to clumping of HA especially in the lips. Massaging the area immediately following injection is the best way to prevent lumps from persisting. It is important to have patients understand the expected clinical course of swelling, firmness, and then softening which typically occurs over the course of 1 week. One of the benefits of using HA for the less experienced user is the fact that they can be readily broken down by the hyaluronidase.

\section{Biodegradeable microparticle injectible implants Calcium hydroxylapatite}

This particular augmenting agent contains smooth spheres of (30\%) calcium hydoxylapetite between 25 and 45 microns and $70 \%$ carboxymethylcellulose gel suspension. This gives the product a white color which is then injected subdermally typically in a threading technique using a unique 28-gauge needle with a foraminal diameter of 27 -gauge. This filler is also unique in that the standard syringe volume is $1.5 \mathrm{~mL}$ of material, making it the largest packaged syringe by volume. Radiesse has been cleared by the FDA for correction of lipoatrophy in persons with human immunodeficiency virus. ${ }^{15}$ In 2006, a cosmetic approval was granted for the correction of moderate to severe wrinkles and folds. Although it can appear radiopaque in radiographic films, there is no indication that it causes masking of abnormal tissues. ${ }^{15}$ It is important to stress that there have been no reported cases of granulomas in over 1000 patients treated with Radiesse with follow up for 5 years. Its persistence in clinical effectiveness is greater when compared to hyaluronic gels in the nasolabial grooves, and for many physicians it is becoming a first line of choice in soft tissue augmentation. ${ }^{16,17}$ Radiesse is not a mucopolysaccharide and therefore does not rely on water binding for its persisting clinical effect and it does not carry the risk of producing Tyndall effect (blue-gray discoloration) in the skin. Because it contains microspheres of calcium hydroxylapatite and collagen forms around these particles (Figure 5), these physical properties can lead to more palpability in the soft subcutaneous tissues of the lips. Therefore Radiesse is not recommended for use in the pink body of the lips to avoid palpability or nodule formation. Treatment of nodules can be reversed by a slit incision and surgical removal as they are very well circumscribed and do 
not produce significant inflammation beyond their physical boundary. It is important to stress that no true granulomas have been seen with the use of Radiesse as all of the material is biocompatible.

\section{Poly-L-lactic acid (PLLA)}

Another injectible implant with micoparticles is Sculptra. It is supplied as a vial $(367.5 \mathrm{mg})$ of freeze dried powder of synthetic L-polymer of polylactic acid (which is from the alpha-hydroxy-acid family), sodium carboxymethylcellulose, and mannitol. This vial must be reconstituted with sterile water at a minimum of 2 hours prior to use but in clinical practice is usually done 1 day prior to its use. ${ }^{18}$ Sculptra was approved by the FDA in 2004 for correction of HIV-related facial atrophy. This compound had been known as New-Fill in Europe and has been available since 1999. It is currently undergoing review for possible esthetic clearance by the FDA. The method of volume enhancement with this particular product is thought to be due to a controlled inflammation where fibroblasts leave collagen as the PLLA is degraded. Clinical results are typically seen after 4 to 6 weeks as the immediate swelling from the diluent resolves after 48 hours.

The esthetic success with this product in immunocompetent patients is dependent on dilution volume and its correct placement. The original recommended dilution volume of $3 \mathrm{~mL}$ had a high incidence $(30 \%-52 \%)$ of subcutaneous nodules which most of the time were palpaple but not visible. ${ }^{19}$ As more volume of diluent $(5 \mathrm{~mL}$ is now the minimum recommended volume per vial) has been added, the incidence of nodules has dropped to $6 \%$ to $13 \%$. $^{20}$ It is also important to allow enough time for the diluents of sterile water to adequately hydrate the particles and therefore proper planning is required for patient appointments. The microparticles of PLLA need to be placed subdermally at subcutaneous junction with a minimum of a 26-gauge needle to avoid clogging. (Figure 6). One must massage the injection sites immediately afterwards and have the patient continue to massage for 5 days post-treatment. It is now recommended to use 5 to $6 \mathrm{~mL}$ of diluents with adequate set up time to help diminish the incidence of nodules. Sculptra can be delivered through a depot method or a crosshatching linear threading technique in the cheeks, temples, and lateral face. Sculptra should not be injected in the periorbital area or the lips because palpable and visible nodules in these areas are difficult to treat. Either technique or combinations has given patients persistence of volume that have lasted for up to 2 years. $^{20}$

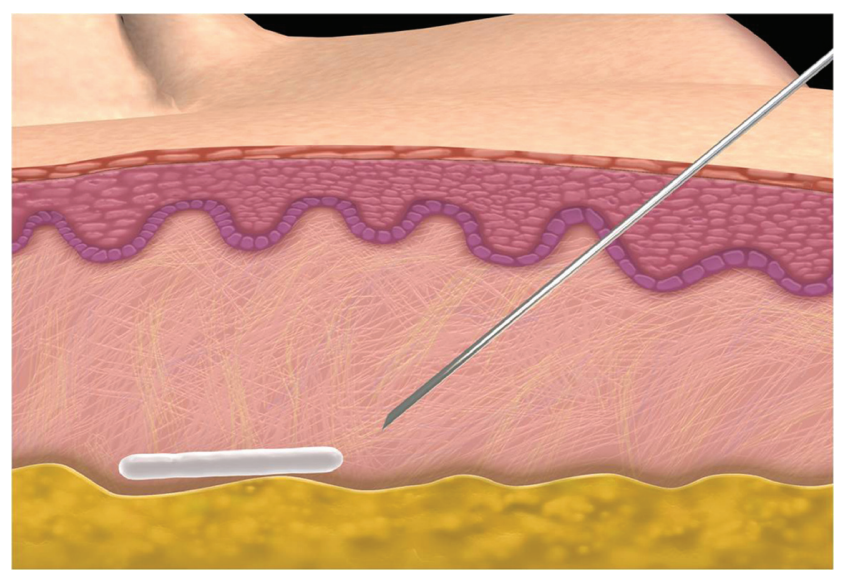

Figure 6 Illustration showing proper placement of poly-L-lactic acid (Sculptra) in the deep dermis/subcutaneous fat. Top layer $=$ epidermis, Middle layer $=$ dermis, Bottom layer in yellow $=$ subcutaneous fat.

\section{Non-absorbable fillers}

\section{Artefill (poly methylmethacryalate PMMA with collagen)}

Within the US, there is limited experience with permanent fillers. The only FDA approved product is Artefill (Artes Medical) which is a combination of bovine collagen and particles of polymethylmethacralate (PMMA). PMMA consists of non-absorbable microspheres 20 to 50 microns in diameter. The carrier gel is composed of 3.5\% bovine collagen, $92.6 \%$ buffered isotonic water, $0.3 \%$ lidocaine $\mathrm{HCl}, 2.7 \%$ phosphate buffer, and $0.9 \%$ sodium chloride which is resorbed over 2 to 3 months. After resorption of the bovine collagen and gel carrier, the resulting PMMA microspheres are surrounded by neocollagen. Skin testing is needed for Artefill because of the bovine collagen component. This filler is injected with a 26-gauge needle that is supplied and should not be overcorrected. Because of the smooth microspheres and the filtered size of PMMA (20-51 microns), with Artefill there was a very low incidence of granuloma which plagued earlier formulations of other products that contained PMMA. ${ }^{21}$ Our limited use of this product over the past year has yielded good results in the marionette area and lateral commissure lines with a series of 2 to 3 injections over 6 to 8 months. At the end of 2008, Artes Medical ceased operations in the US and the fate of this particular product remains unclear as of the time of writing this paper.

\section{Silicone}

Liquid silicone fine droplet injection is used on a limited basis by certain physicians for long-term permanent corrections, but with significant risks. ${ }^{22}$ As this is a permanent material, correction of problems usually requires surgical excision. The risk of granuloma formation has always existed which 
has been attributed to a number of factors including purity, particle size, and injection technique. There is no approved silicone for soft tissue skin augmentation. However, off-label uses of ophthalmologic preparations (AdatoSil ${ }^{\circledR} 5000$ and Silikon $\left.{ }^{\circledR} 1000\right)$ of silicone have been adapted for use in the treatment of HIV-associated-lipoatrophy and other clinical conditions of facial volume loss. Some studies are underway to evaluate Silskin (a liquid silicone similar to Silikon 1000) using the microdroplet technique which involves small aliquots 0.01 to $0.03 \mathrm{~mL}$ spaced at 2 to $10 \mathrm{~mm}$ distances in the subcutaneous tissues. ${ }^{23}$ While silicone has great promise as a permanent filler, there is much to be analyzed from the current and future studies.

\section{Summary}

The current options for soft tissue augmentation have increased because of the safety and differentiation of the injectible products. While skeletal onlay grafts and autologous fat augmentation have helped surgeons address most soft tissue deficiencies, we now see the use of additional fillers giving patients and non surgeons options for achieving temporary enhancement. The current filler choices can add to further soft tissue replacements as patients age with the more standard skeletal onlay grafts. The development of the saline lip implant serves as an example of how alloplastic materials attempt to mimic more the viscoelastic properties of natural soft tissue without the need for repeated treatments as with temporary fillers. There is no doubt that the use of soft tissue fillers will become more widespread as they have become well liked by patients and physicians.

\section{Disclosures}

The authors report no conflicts of interest in this work.

\section{References}

1. Binder WJ, Bloom DC. The use of custom designed midfacial and submalar implants in the treatment of facial wasting syndrome. Arch Facial Plast Surg. 2004;6:394-397.

2. Coleman SR. Structural fat grafts: the ideal filler? Clin Plast Surg. 2001;28:111-117.
3. Lamperti TA, Carithers JS. Subcutaneous superficial musculoaponeurotic system grafting of the aging melolabial furrow. Arch Facial Plast Surg. 2004;6:384-388.

4. Niamtu J. Experience and evaluation of a new saline filled implant for cosmetic lip enhancement. Am J Cosmet Surg. 2007;4:1-10.

5. Narins RB, Brandt F, Lyeden J, Lorenc ZP, Rubin M, Smith B. A randomized doubleblind multicenter comparison of the efficacy and tolerability of Restylane versus Zyplast for the correction of nasolabial folds. Dermatol Surg. 2003;29:588-595.

6 Evolence $^{\circledR}$ [package insert]. OrthoNeutrogena, Summit, NJ, USA.

7 Larsen NE, Pollak CT, Reiner K, Leschiner E, Balazs EA. Hylan gel biomaterial: dermal and immunologic compatibility. J Biomed Materials Res. 1993;27:1129-1134.

8. Falcone SJ, and Berg RA. Cross-linked hyaluronic acid dermal fillers: a comparison of rheological properties. J Biomed Mater Res A. 2008 Jan $15 ; 18200557$.

9. Wang F, Garza LA, Kang S, et al. In vivo stimulation of de novo collagen production caused by cross-linked hyaluronic acid dermal filler injections in photodamaged human skin. Arch Dermatol. 2007;143: $155-163$.

10. Elevess ${ }^{\circledR}$ [package insert]. Anika Pharmaceuticals, Waltham, MA, USA.

11. Prevelle ${ }^{\circledR}$ Silk [package insert]. Genzyme, USA

12. Restylane ${ }^{\circledR}$ [package insert]. Medicis Aesthetics, Scottsdale, AZ, USA

13. Perlane ${ }^{\circledR}$ [package insert]. Medicis Aesthetics, Scottsdale, AZ, USA.

14. Juvederm ${ }^{\circledR}$ [package insert]. Allergan, Irvine, CA, USA.

15. Tzikas, TL. Evaluation of Radiance FN soft tissue filler for facial soft tissue augmentation. Arch Facial Plast Surg. 2004;6234-6239.

16. Moers-Carpi M, Vogt S, Santos G, Planas J, Vallve S, Howell D. A multicenter randomized trial comparing calcium hydroxlyapetite to two hyaluronic acids for the treatment of nasolabial folds. Dermatol Surg. 2007;33:S144-S151.

17. American Society of Plastic Surgeons 2000/2006/2007 National Plastic Surgery Statistics. Cosmetic and Reconstructive Procedure Trends. 2007.

18. Sculptra ${ }^{\circledR}$ [package insert]. Sanofi-Aventis, Summit, NJ, USA.

19. Valantin MA, Aubron-Olivier C, Ghosn J, et al. Polylactic acid implants (New-Fill) to correct facial lipoatrophy in HIV-infected patients: results of the open-label study VEGA. AIDS. 2003;17:2471-2477.

20. Vleggaar D. Soft tissue augmentation and the Role of poly 1-lactic acid. Plast Reconstr Surg. 2006;118:46-54.

21. Alcalay J, Alkalay R, Gat A, Yorav S. Late-onset granulomatous reaction to Artecoll. Dermatol Surg. 2003;29:859-862.

22. Rapaport MJ, Vinnik C, Zarem H. Injectable silicone: cause of facial nodules, cellulitis,ulceration, and migration. Aesthetic Plast Surg. 1996;20:267-276.

23. Narins RS, Beer K. Liquid Injectible silicone: a review of its history, immunology, technical considerations complications and potential. Plast Reconstru Surg. 2006;118(S):77-84.
Clinical, Cosmetic and Investigational Dermatology

\section{Publish your work in this journal}

Clinical, Cosmetic and Investigational Dermatology is an international, peer-reviewed, open access, online journal that focuses on the latest clinical and experimental research in all aspects of skin disease and cosmetic interventions. All areas of dermatology will be covered; contributions will be welcomed from all clinicians and

\section{Dovepress}

basic science researchers globally. This journal is indexed on CAS The manuscript management system is completely online and includes a very quick and fair peer-review system, which is all easy to use. Visit http://www.dovepress.com/testimonials.php to read real quotes from published authors. 